\title{
Presença de Aelurostrongylus abstrusus em amostras fecais de gatos no município de Pelotas, RS, Brasil
}

\author{
Presence of Aelurostrongylus abstrusus in faecal samples of cats in the \\ municipality of Pelotas, RS, Brazil
}

\begin{abstract}
Alexsander Ferraz (1)*, Bruna dos Santos Pires (D), Eduarda Machado dos Santos, Eugênia Tavares Barwaldt (D), Paola Renata Joanol Dallmann (D), Carolina da Fonseca Sapin (D), Camila Moura de Lima (D), Diego Moscarelli Pinto (1) , Márcia de Oliveira Nobre (1), Leandro Quintana Nizoli (1)
\end{abstract}

Universidade Federal de Pelotas (UFPel), Pelotas, RS, Brasil

\section{Resumo}

Aelurostrongylus abstrusus é um nematódeo que acomete o trato respiratório dos felinos, sendo considerado o parasito mais comum do parênquima pulmonar dos gatos. Pelo fato de os sinais clínicos serem inespecíficos, o uso de técnicas coproparasitológicas é importante para o diagnóstico definitivo. Dentre estas, a técnica de Baermann é considerada a de eleição. Este trabalho teve como objetivo avaliar a presença de larvas de A. abstrusus em amostras fecais de gatos domésticos do município de Pelotas, Rio Grande do Sul, Brasil. Foram analisadas, através da técnica de Baermann, 60 amostras. Destas, duas foram positivas para larvas de $1^{\circ}$ estágio deste parasito, representando 3,3\% do total. Estes dados evidenciam a importância da realização desta técnica no diagnóstico de aelurostrongilose, sendo relevante para o diagnóstico diferencial das afecções respiratórias dos felinos.

Palavras-chave: Fezes. Diagnóstico. Parasito pulmonar. Gatos.

\begin{abstract}
Aelurostrongylus abstrusus is a nematode that affects the respiratory tract of cats, being considered the most common parasite of the pulmonary parenchyma of cats. Because the clinical signs are nonspecific, the use of coproparasitological techniques is important for the definitive diagnosis. Baermann's technique is considered
\end{abstract}


the one of choice. The objective of this work was to evaluate the presence of A. abstrusus larvae in faecal samples of domestic cats of the municipality of Pelotas, Rio Grande do Sul, Brazil. Sixty samples were analyzed using Baermann technique. Of these, two were positive for first stage larvae of this parasite, representing 3.3\% of the total. These data show the importance of performing this technique in the diagnosis of aelurostrongylosis, being relevant for the differential diagnosis of feline respiratory pathologies.

Keywords: Feces. Diagnosis. Pulmonar parasite. Cats.

\section{Introdução}

O número de felinos como animais de estimação vem crescendo nos últimos anos, em virtude da personalidade e do modo como se comportam, adaptando-se facilmente à rotina da população moderna (Genaro, 2010; Lima e Luna, 2012). Desta forma, houve um aumento no interesse pelas doenças que acometem esta espécie, como é o caso da aelurostrongilose, também conhecida por estrongilose cardiopulmonar do gato (Silva et al., 2005; Traversa et al., 2010; Elsheikha et al., 2016; Penagos-Tabares et al., 2018).

Aelurostrongylus abstrusus, helminto da família Angiostrongylidae, é o principal parasito pulmonar de felinos (Silva et al., 2005; Barutzki e Schaper, 2013; Diakou et al., 2015). É um nematódeo capaz de provocar pneumonia verminótica, de gravidade variável, independentemente de raça e sexo, não sendo considerada uma zoonose (Teixeira et al., 2008; Mircean et al., 2010; Elsheikha et al., 2016). Gatos jovens e que vivem de forma peridomicilar ou errante têm maior probabilidade de ficarem infectados, devido ao comportamento de caça e por estarem mais suscetíveis a ingerir hospedeiros intermediários e paratênicos (Mircean et al., 2010; Knaus et al., 2011; Barutzki e Schaper, 2013; Jeżewski et al., 2013; Riggio et al., 2013).

O ciclo biológico do $A$. abstrusus é indireto e envolve moluscos como hospedeiros intermediários, bem como aves, répteis, anfíbios e pequenos mamíferos como hospedeiros paratênicos (PayoPuente et al., 2008; Olsen et al., 2015). O gato doméstico é infectado pela ingestão destes hospedeiros. As larvas infectantes de terceiro estágio (L3) penetram na mucosa do trato digestório e seguem para os pulmões através da circulação sanguínea ou linfática, onde se desenvolvem até as formas adultas (Ribeiro e Lima, 2001; Olsen et al., 2015).

Os vermes adultos se localizam nos bronquíolos terminais e nos ductos alveolares, onde os ovos são depositados pelas fêmeas adultas (PayoPuente et al., 2008; Elsheikha et al., 2016). As larvas de primeiro estágio (L1) eclodem e, como têm mobilidade própria, movimentam-se ativamente dos bronquíolos terminais até a parte superior do trato respiratório, onde são deglutidas e posteriormente eliminadas para 0 ambiente através das fezes (Rassoui et al., 2015; Elsheikha et al., 2016). As larvas L1 penetram nos hospedeiros intermediários onde se desenvolvem até L3, que é a forma infectante para os gatos (Ribeiro e Lima, 2001; Olsen et al., 2015).

O período pré-patente varia geralmente entre cinco a seis semanas e a duração da patência é de cerca de quatro meses (Traversa et al., 2008; Elsheikha et al., 2016). Alguns parasitos adultos, entretanto, podem sobreviver vários anos nos pulmões, apesar da ausência de larvas nas fezes (Bowman, 2003; Schnyder et al., 2014).

As manifestações clínicas dependem da carga parasitária, idade, resposta imune do animal e presença de doenças concomitantes (Silva et al., 2005; Elsheikha et al., 2016). Geralmente, a enfermidade tem caráter crônico e, na ausência de uma elevada carga parasitária, a infecção é espontaneamente eliminada entre três a quatro meses; após a eliminação, o felino fica resistente à reinfecção (Ribeiro e Lima, 2001; Elsheikha et al., 2016).

O diagnóstico pode ser realizado através de exames coproparasitológicos (Tüzer et al., 2002; Grandi et al., 2005; Conboy, 2009), lavado traqueal (Barrs, 1999; Ribeiro et al., 2014), histologia (Willard, 1988) e radiografia de pulmão (Losonsky, 1983; Willard, 1988; Barrs, 1999). Todavia, a técnica de eleição para o diagnóstico do parasito $A$. abstrusus é o método de Baermann, que consiste em um teste qualitativo direto (Hoffmann, 1987; Barutzki e Schaper, 2013; Elsheikha et al., 2016). 
O objetivo deste trabalho foi determinar a presença de larvas de $A$. abstrusus em amostras de fezes de gatos domiciliados no município de Pelotas, Rio Grande do Sul, Brasil.

\section{Material e métodos}

Foram processadas e analisadas amostras fecais de 60 gatos domiciliados no município de Pelotas (3146'19"S, 52²0'33"W). Destes, 43 $(71,7 \%)$ eram adultos e $17(28,3 \%)$ eram filhotes. As coletas foram realizadas pelos próprios tutores, acondicionadas em potes plásticos, mantidas sob refrigeração e encaminhadas para o Laboratório de Doenças Parasitárias (Ladopar) da Faculdade de Veterinária da Universidade Federal de Pelotas (FAVet/UFPel), durante o ano de 2018.

A técnica adotada para a análise do material foi a de Baermann (1917), utilizada para pesquisa de larvas. A leitura das lâminas foi realizada em microscópio óptico, com objetivas de 100 e 400x. As amostras foram consideradas positivas quando identificadas como L1. A confirmação se deu pelas características morfológicas da larva, pois esta apresenta a cauda recurvada, dotada de espinho subterminal.

\section{Resultados e discussão}

Das amostras analisadas, constatou-se que duas $(3,3 \%)$ estavam positivas para larvas de $A$. abstrusus (Figura 1), sendo uma encontrada em felino adulto e a outra em filhote, ambos com histórico de tosse recorrente.

Alguns estudos têm sido realizados para detectar a presença deste parasito em amostras de fezes de gatos. Melo Jr et al. (2016), estudando a ocorrência de endoparasitos em 50 amostras fecais de gatos no município de Patos de Minas, MG, encontraram $2 \%$ de positividade para este parasito, valor um pouco abaixo ao observado no presente estudo. Frequência mais alta foi encontrada por Ehlers et al. (2013) que, analisando a prevalência de $A$. abstrusus em gatos no município de Porto Alegre, RS, obtiveram uma taxa de $29,5 \%$ de amostras positivas (24/88).

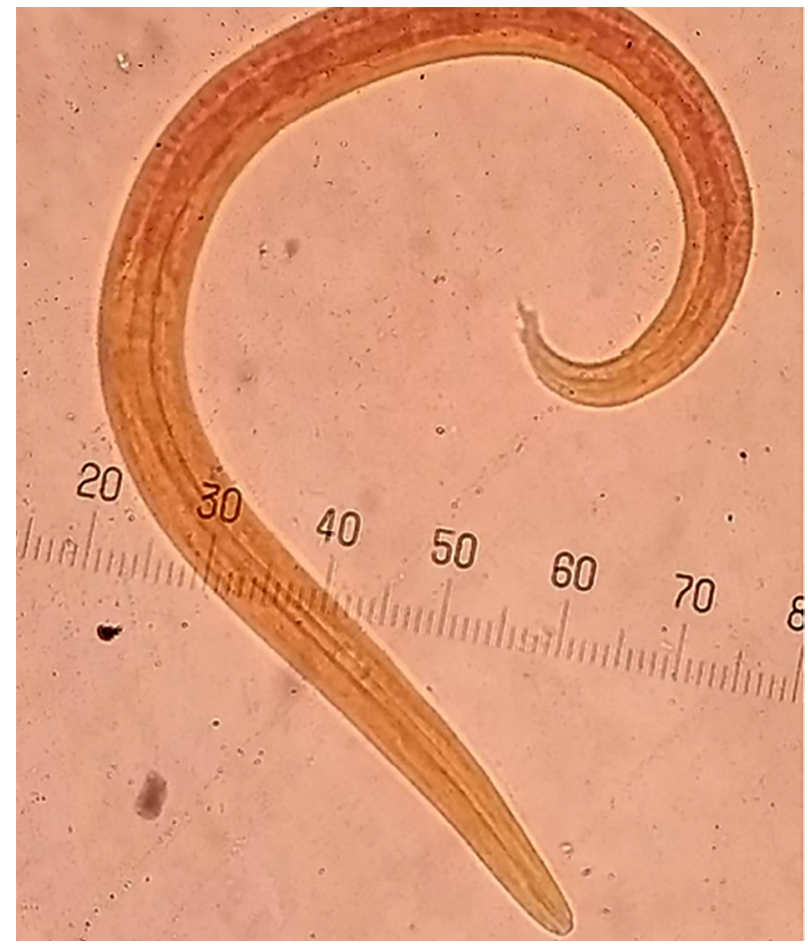

Figura 1 - Larva de $1^{\circ}$ estágio de $A$. abstrusus em felino, apresentando a cauda recurvada dotada de espinho subterminal.

É importante ressaltar que alguns animais parasitados por $A$. abstrusus são assintomáticos, devido à forma subclínica e evolução autolimitante da doença (Elsheikha et al., 2016). Nas infecções moderadas, observam-se alterações como tosse, espirros e secreção nasal mucopurulenta (Bowman, 2003; Barutzki e Schaper, 2013; Schnyder et al., 2014). Nos casos graves ou quando o animal está debilitado e/ou imunodeprimido, verificamse tosse, dispneia, polipneia, letargia e perda de peso, podendo a doença ser fatal (Tüzer et al., 2002; Bowman, 2003; Grandi et al., 2005; Barutzki e Schaper, 2013).

Pelo fato de os sinais clínicos apresentados serem inespecíficos, comuns a afecções respiratórias de diferentes etiologias, o diagnóstico clínico é difícil, sendo recomendada a realização de exames complementares para o diagnóstico definitivo, como o coproparasitológico. É importante, porém, que o método parasitológico a ser empregado seja adequado para a pesquisa de larvas (Mello et al., 2000). 
A técnica de Baermann (1917) é considerada a de eleição para diagnóstico de aelurostrongilose, pois ela permite a recuperação de larvas com termohidrotropismo positivo, como as do $A$. abstrusus (Traversa et al., 2008; Lacorcia et al., 2009). Como a eliminação das larvas nas fezes pode ocorrer de forma intermitente, recomendase a realização de exames seriados para aumentar a sensibilidade do teste (Carvalho et al., 2002). Esta intermitência pode justificar a presença de apenas dois animais positivos no presente estudo, podendo existir entre as amostras analisadas animais falso-negativos.

\section{Conclusão}

A partir dos resultados encontrados, fica evidente a importância da realização de estudos para determinar a prevalência do parasito $A$. abstrusus e da utilização da técnica de Baermann no diagnóstico de aelurostrongilose, sendo fundamental para o diagnóstico diferencial das afecções respiratórias dos felinos. No entanto, apesar do seu baixo custo, eficácia e simplicidade, esta técnica continua a ser pouco utilizada na prática clínica de animais de companhia. Estimase, por isso, que a verdadeira prevalência destas parasitoses esteja subestimada no Brasil.

\section{Referências}

Baermann G. Eine einfache Methode zur Auffindung von Ankylostomum (Nematoden) Larven in Erdproben. Batavia, Indonésia: Javasche Boekhandel \& Drukkerij; 1917. p. 41-7.

Barrs VR, Swinney GR, Martin P, Nicoll RG. Concurrent Aelurostrongylus abstrusus infection and salmonellosis in a kitten. Aust Vet J. 1999;77(4):229-32.

Barutzki D, Schaper R. Occurrence and regional distribution of Aelurostrongylus abstrusus in cats in Germany. Parasitol Res. 2013;112(2):855-61.

Bowman DD. Georgis' Parasitology For Veterinarians. 8 ed. Filadélfia: Saunders; 2003. 432 p.
Carvalho FM, Falcão AO, Albuquerque MC, Silva P, Bastos OMP, Uchôa CMA. Diagnóstico coproparasitológico: estudo comparativo entre os métodos de Faust \& cols., Lutz, Baermann \& Moraes e Coprotest ${ }^{\circledR}$. Rev Bras Anal Clin. 2002;34(2):75-7.

Conboy G. Helminth parasites of the canine and feline respiratory tract. Vet Clin North Am Small Anim Pract. 2009;39(6):1109-26.

Diakou A, Di Cesare A, Barros LA, Morelli S, Halos L, Beugnet $F$, et al. Occurrence of Aelurostrongylus abstrusus and Troglostrongylus brevior in domestic cats in Greece. Parasit Vectors. 2015;8:590.

Ehlers A, Mattos MJT, Marques SMT. Prevalência de Aelurostrongylus abstrusus (Nematoda, Strongylida) em gatos de Porto Alegre, Rio Grande do Sul. Rev FZVA. 2013;19(1):97-104.

Elsheikha HM, Schnyder M, Traversa D, Di Cesare A, Wright I, Lacher DW. Updates on feline aelurostrongylosis and research priorities for the next decade. Parasit Vectors. 2016;9(1):389.

Genaro G. Gato doméstico: futuro desafio para controle da raiva em áreas urbanas? Pesq Vet Bras. 2010;30(2):186-9.

Grandi G, Calvi LE, Venco L, Paratici C, Genchi C, Memmi D, et al. Aelurostrongylus abstrusus (cat lungworm) infection in five cats from Italy. Vet Parasitol. 2005;134(1-2):177-82.

Hoffmann RP. Diagnóstico de parasitismo veterinário. Porto Alegre: Sulina; 1987. 156 p.

Jeżewski W, Buńkowska-Gawlik K, Hildebrand J, PerecMatysiak UM, Laskowski Z. Intermediate and paratenic hosts in the life cycle of Aelurostrongylus abstrusus in natural environment. Vet Parasitol. 2013;198(3-4):401-5.

Knaus M, Kusi I, Rapti D, Xhaxhiu D, Winter R, Visser M, et al. Endoparasites of cats from the Tirana área and the first report of Aelurostrongylus abstrusus (Railliet, 1898) in Albania. Wien Klin Wochenschr. 2011;123(Suppl 1):31-5.

Lacorcia L, Gasser RB, Anderson GA, Beveridge I. Comparison of bronchoalveolar lavage fluid examination and other diagnostic techniques with the Baermann 
technique for detection of naturally occurring Aelurostrongylus abstrusus infection in cats. J Am Vet Med Assoc. 2009;235(1):43-9.

Lima AFM, Luna SPL. Algumas causas e consequências da superlotação canina e felina: acaso ou descaso? Rev mv\&z. 2012;10(1):32-8.

Losonsky JM, Thrall DE, Prestwood AK. Radiographic evaluation of pulmonary abnormalities after Aelurostrongylus abstrusus inoculation in cats. Am J Vet Res. 1983;44(3):478-82.

Melo Jr FF, Cunha GN, Bombonato NG, Guimarães EC. Ocorrência de endoparasitos em gatos (Felis catus Linnaeus, 1758) no município de Patos de Minas, MG. Anais $37^{\circ}$ Congresso Brasileiro da ANCLIVEPA; 12-14 mai 2016; Goiânia, GO. p. 133-7.

Mello RT, Rocha MO, Moreira MCCG. Exame coproparasitológico de fezes: estudo comparativo entre os métodos Coprotest, MIFC, Baermann e Kato. Rev Bras Anal Clin. 2000; 32(4):289-91.

Mircean V, Titilincu A, Vasile C. Prevalence of endoparasitas in household cat (Felis catus) populations from Transylvania (Romania) and association with risk factors. Vet Parasitol. 2010;171(1-2):163-6.

Olsen CS, Willesen JL, Pipper CB, Mejer H. Occurrence of Aelurostrongylus abstrusus (Railliet, 1898) in Danish cats: a modified method for isolating adult worms. Vet Parasitol. 2015;210(1-2):32-9.

Payo-Puente P, Botelho-Dinis M, Urueña AMC, Payo-Puente M, Gonzalo-Orden JM, Rojo-Vazquez FA. Prevalence study of the lungworm Aelurostrongylus abstrusus in stray cats of Portugal. J Feline Med Surg. 2008;10(3):242-6.

Penagos-Tabares F, Lange MK, Chaparro-Gutiérrez JJ, Taubert A, Hermosilla C. Angiostrongylus vasorum and Aelurostrongylus abstrusus: Neglected and underestimated parasites in South America. Parasit Vectors. 2018;11:208.

Rassoui M, Ghaderi J, Goudarzi A, Sbouri S. Aelurostrongylus abstrusus in a stray cat's blood stream. Comp Clin Pathol. 2015;24(4):773-5.
Ribeiro VM, Barçante JMP, Negrão-Corrêa D, Barçante TA, Klein A, Lima WS. Lavado broncoalveolar como ferramenta para avaliação da alteração celular durante a infecção por Aelurostrongylus abstrusus em gatos. Pesq Vet Bras. 2014;34(10):990-5.

Ribeiro VM, Lima WS. Larval production of cats infected and re-infected with Aelurostrongylus abstrusus (Nematotoda: Protostrongylidae). Revue Med Vet. 2001; 152(11):815-20.

Riggio F, Mannella R, Ariti G, Perrucci S. Intestinal and lung parasites in owned dogs and cats from central Italy. Vet Parasitol. 2013;193(1-3):78-84.

Schnyder M, Di Cesare A, Basso W, Guscetti E, Riond B, Glaus $T$, et al. Clinical, laboratory and pathological findings in cats experimentally infected with Aelurostrongylus abstrusus. Parasitol Res. 2014;113(4):1425-33.

Silva JMF, Fonseca IMP, Carvalho LMM, Meireles JAFS, Fazendeiro I. Pneumonia em gato por Aelurostrongylus abstrusus - necessidade de um diagnóstico precoce. Rev Port Cienc Vet. 2005;100(553-4):103-6.

Teixeira MC, Velloso TF, Fialho CG, Marques ST, Escopelli K, Araújo FAP. Aelurostrongylus abstrusus: relato de caso em felino. Rev Clin Vet. 2008;74:52-4.

Traversa D, Lia RP, lorio R, Boari A, Paradies P, Capelli $\mathrm{G}$, et al. Diagnosis and risk factors of Aelurostrongylus abstrusus (Nematoda, Strongylida) infection in cats from Italy. Vet Parasitol. 2008;153(1-2):182-6.

Traversa D, Di Cesare A, Conboy G. Canine and feline cardiopulmonary parasitic nematodes in Europe: emerging and underestimated. Parasit Vectors. 2010;3:62.

Tüzer E, Toparlak M, Gargili A, Keles V, Esatgil MU. A case of Aelurostrongylus abstrusus infection in a cat in Istanbul, Turkey and its treatment with moxidectin and levamisole. Turk J Vet Anim Sci. 2002;26(2):411-4.

Willard MD, Roberts RE, Allison N, Grieve RB, Escher K. Diagnosis of Aelurostrongylus abstrusus and Dirofilaria immitis infectios in cats from a humane shelter. J Am Vet Med Assoc. 1988;192(7):913-6. 